\section{No depot for alum}

Alum is the most widely used clinical adjuvant, but its mode of action remains contentious, although the formation of depots for the slow release of antigen is often cited as one of its main immunostimulatory mechanisms. In the FASEB Journal, Brewer and colleagues use an approach of trangenic expression of a $\mathrm{T}$ cell antigen receptor and the model antigen EaGFP to rule out the of the possiblity that the formation of a depot of alum is responsible for its adjuvanticity. After antigen priming with alum or the Tolllike receptor agonist $\mathrm{CpG}$, which is an adjuvant but cannot form a depot, they find no difference in the kinetics or magnitude of the $\mathrm{T}$ cell response or in the delivery of antigen to a variety of antigenpresenting cells. Alum forms discrete and removable foci at its injection site, and excision of these foci, even as early as 2 hours after injection, has no effect on either primary or recall responses. Rather than being adsorbed to the alum, antigen is therefore rapidly disseminated in soluble form.

$Z F$

FASEB J. 26, 1272-1279 (2012)

\section{STING scaffold}

STING, a transmembrane protein in the endoplasmic reticulum, interacts with various cytosolic DNA sensors to induce the production of interferon- $\beta$ via a pathway dependent on the transcription factor IRF3. In Science Signaling, Tanaka and Chen show that STING acts as a scaffold that recruits IRF3. This interaction requires Ser366 and Leu374 in the conserved carboxy-terminal domain of STING. This domain also recruits and activates the kinase TBK1. TBK1 phosphorylates IRF3, which leads to its dimerization and translocation to the nucleus. Notably, although TBK1 is activated by many signaling pathways, such as Toll-like receptors, not all lead to IRF3 activation. In response to cytosolic duplex DNA, however, STING is needed to place IRF3 in proximity with TBK1, thereby activating IRF3-dependent responses to combat infection with double-stranded DNA viruses or intercellular bacteria.

$L A D$ Sci. Signal. 5, 214 ra20 (2012)

\section{Suppression by Helicobacter}

The gut bacterium Helicobacter pylori can cause gastritis and cancer when introduced into adults but can be pro-tolerogenic in neonates. In the Journal of Clinical Investigation, Müller and colleagues identify the mechanism by which $H$. pylori infection can engender tolerance. Both in vivo and in vitro, H. pylori suppresses the activation of dendritic cells (DCs) in a contactdependent way, which leads to a 'semi-mature' state. These semi-mature DCs are poor antigen-presenting cells and also convert naive effector cells into Foxp $3^{+}$regulatory $T$ cells $\left(T_{\text {reg }}\right.$ cells). These $T_{\text {reg }}$ cells in turn suppress an experimental model of asthma, a finding consistent with some results obtained with humans showing amelioration of autoimmune disease associated with $\boldsymbol{H}$. pylori infection. The ability of DCs exposed to $\boldsymbol{H}$. pylori to elicit $\mathrm{T}_{\text {reg }}$ cells is entirely dependent on their production of interleukin 18 and intact signaling via its receptor in the target T cells.

J. Clin. Invest. 122, 1082-1096 (2012)

\section{Stealthy silencing}

After being infected, host cells upregulate many genes encoding molecules involved in immune defense to combat the invading microbe. In Nature, Tarakhovsky and colleagues identify a unique strategy used by $\mathrm{H} 3 \mathrm{~N} 2$ influenza A virus to evade the immune response. The nonstructural protein NS1 of $\mathrm{H} 3 \mathrm{~N} 2$, which accumulates in the nucleus during infection, has a carboxy-terminal domain that acts as a histone mimic that can be methylated or acetylated similarly to Lys4 of histone H3. Methylated NS1 binds to and sequesters the transcriptional-elongation regulator hPAF1 in human cells. Genome-wide profiling shows accumulation of wild-type NS1 on the promoters of induced antiviral genes, which blocks their full-length transcription. Human cells infected with wild-type $\mathrm{H} 3 \mathrm{~N} 2$ produce higher viral titers than do those infected with H3N2 variants that express NS1 lacking the hPAF1-interaction site. The H3N2 NS1 histone mimic thereby selectively alters inducible gene expression to subvert host antiviral defenses.

$L A D$

Nature (14 March 2012) doi:10.1038/nature10892

\section{The hygiene hypothesis}

Epidemiologic observations suggest that exposure to microbes in early life is associated with the prevention of diseases such as inflammatory bowel disease and asthma. In Science, Blumberg and colleagues show that more invariant natural killer cells (iNKT cells) accumulate in the lungs and colonic lamina propria and result in greater susceptibility to inflammatory bowel disease and asthma in germ-free mice than in specific pathogen-free mice. Exposure of neonatal germ-free mice to conventional microbiota normalizes the number of iNKT cells and results in less susceptibility to induced disease, but exposure of adult germ-free mice does not. The colons and lungs of adult germ-free mice have higher expression of CXCL16, a chemotactic factor for $i$ KKT cells. Specific regions in Cxcl16 are hypermethylated in a tissue-specific way in germ-free mice, and colonization of neonatal mice with conventional microbiota reverses this epigenetic modification, but colonization of adult mice does not. The mechanisms by which the microbiota regulates CXCL16 expression and thus the accumulation of $i$ NKT cells are unknown.

Science (22 March 2012) doi:10.1126/science.1219328

\section{Thymus dialogs}

Medullary thymic epithelial cells (mTECs) that express the autoimmune regulator Aire are specialized cells that regulate thymocyte selection and are essential for establishing tolerance to self. In Immunity, Anderson and colleagues show that progenitors of $\mathrm{V}_{\gamma} 5^{+}$dendritic epidermal $\mathrm{T}$ cells (DETCs) are involved in the maturation of Aire ${ }^{+}$mTECs in embryonic thymus. $\mathrm{V}_{\gamma} 5^{+}$DETC progenitors express the ligand for the cytokine receptor RANK and control the maturation of Aire ${ }^{+}$mTECs in a RANKdependent way. In turn, Aire ${ }^{+}$mTECs foster the maturation of $\mathrm{V}_{\gamma} 5^{+}$ DETC progenitors dependent on the selection molecule Skint-1 but independent of Aire. These results suggest that before the emergence of positively selected $\alpha \beta$ thymocytes, which are a source of RANK ligand and contribute to the maintenance of Aire ${ }^{+}$mTECs in the adult thymus, the first embryonic waves of $\gamma \delta$ T cell progenitors establish similar reciprocal links with the thymic epithelium.

Immunity 36, 427-437 (2012) 